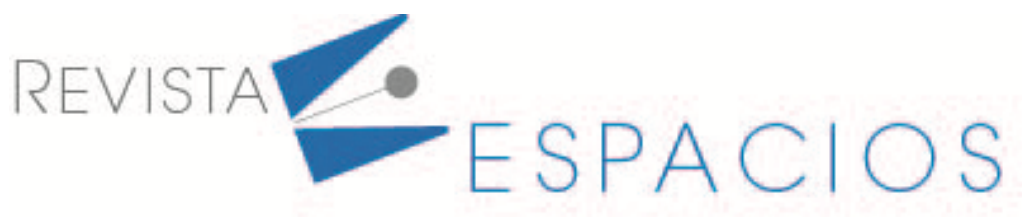

\title{
Tendencias en los procesos de construcción de paz
}

\section{Trends in the peace-building process}

\author{
PEÑA, Parcival ${ }^{1}$ \\ VALERA, Alfonso ${ }^{2}$ \\ MARLES, Claritza ${ }^{3}$
}

\begin{abstract}
Resumen
Con el propósito de identificar tendencias de futuro para la construcción de paz desde la perspectiva de la educación superior, se realizó un estudio en 81 investigaciones, con enfoque interpretativo y las siguientes categorías: construcción de paz, educación superior y escenarios de futuro consultados. Los hallazgos reflejan seis tendencias que evidencian la necesidad de cambio social para la transformación hacia la paz sostenible y de generar condiciones en la construcción de tendencias que contribuyan en la justicia, paz y reconciliación.
\end{abstract}

Palabras Claves: cultura para la paz, escenarios para la construcción de paz, educación.

\begin{abstract}
In order to identify future trends for peace-building from the perspective of higher education, a study was conducted in 80 studies, with an interpretative approach and the following categories:: peace building, higher education and scenarios for the future consulted in 81 research projects. The results reflect six trends that demonstrate the need for social change for the transformation towards sustainable peace and to create conditions in the construction of trends that contribute to justice, peace and reconciliation.
\end{abstract}

Key words: culture for peace, scenarios for peace building, education

\section{Introducción}

Los temas relacionados con la paz y el conflicto exigen de mucha profundidad debido a su complejidad e implicaciones, por ello es necesario identificar los avances significativos en este campo, así como las perspectivas actuales sobre la paz y el conflicto, abordando los orígenes y proyectándolos hacia las coyunturas de paz de carácter nacional como internacional. El propósito de este artículo es identificar las tendencias investigativas de futuro para un proceso de construcción de paz en la educación superior, al considerar que la educación para la paz y el papel de los diferentes actores involucrados es uno de los aspectos relevantes que surgen en un proceso de construcción de paz.

La problemática relacionada con la construcción de paz ha sido abordada desde diversas investigaciones, entre ellas destaca la investigación desarrollada por Nasi y Rettberg (2005), quienes argumentan que el conflicto armado ha generado un fuerte impacto en la sociedad. Uno de estos impactos se refleja en el ámbito académico

\footnotetext{
${ }^{1}$ Candidato a Doctor en Gerencia y Política Educativa. Profesor Investigador. Universidad de la Amazonia. pa.pena@udla.edu.co

2 Doctor en Ciencias Pedagógicas. Profesor Investigador. Universidad de la Habana. orlandovalera@infomed.sld.cu

${ }^{3}$ Candidata a Doctora en Educación y Cultura Ambiental. Profesora investigadora. Universidad de la Amazonia. c.marles@udla.edu.co
} 
mundial, con el número creciente de explicaciones que atribuyen los conflictos y guerras a las confrontaciones bipolares y a las dinámicas políticas, económicas y sociales de carácter interno como externo de regiones o países. Ante esta problemática, en este artículo se abordará el siguiente interrogante: ¿cuáles son las tendencias temáticas en los estudios afines con los procesos de construcción de paz desde la perspectiva de la educación superior?

La construcción de paz es una ruta multifacética y compleja que involucra diversidad de actores con ideales y grados de poder distintos, los cuales generan procesos enmarcados en escenarios y contextos singulares. Por esto, los procesos de construcción de paz presentan avances y retrocesos, conciliaciones parciales y muchas crisis, en gran parte por ser el conflicto un resultado histórico que no permite establecer la paz como un umbral alcanzable, al considerar la paz como una lucha permanente (Nasi y Rettberg, 2005).

La construcción de paz debe ser el resultado del aporte de cada individuo y el acompañamiento de las instituciones que proveen normas y lineamientos políticos que permitan organizar las actuaciones sociales desde el ámbito local hasta el internacional. Además, desde el nivel educativo se deben diseñar metodologías, para integrar las visiones individuales y colectivas que promuevan el consenso y fortalezcan la necesidad de integrar todos los grupos sociales en la búsqueda de una paz sostenible y duradera.

De acuerdo a lo anterior, la apropiación del concepto de paz en una sociedad invita a asumir comportamientos no violentos, para llegar a una transformación social; cada individuo debe interiorizar el significado de paz y aplicarlo día tras día, convirtiéndolo en un hábito, un valor y una manera de relacionarse con su entorno social y cultural, privilegiando el respeto a la vida, la diferencia y la dignidad de cada ser humano como única alternativa para desarrollar una cultura de paz (Jiménez y Jiménez, 2014). Igualmente, Cerdas (2015), considera que para crear una verdadera cultura de paz se hace necesaria una metamorfosis a nivel individual y social, en la que cumple un rol primordial la educación en todos los niveles, siempre desde la perspectiva de los derechos humanos y la solidaridad.

Para Salguero y Seva (2005), el concepto de paz y la creación de cultura de paz exige un modelo educativo con énfasis en la construcción de una sociedad educada con un marcado énfasis en los derechos humanos y el desarrollo humano hacia la paz, por ello se debe incrementar la sensibilidad de los autores sobre los diferentes indicadores de paz para lograr la construcción de una cultura de paz a través de la educación (Torrermorell, Valls, De Nicolás \& Raga, 2014).

Es importante destacar que en el campo de la investigación social se han desarrollado alternativas metodológicas y procedimientos para favorecer la construcción de escenarios de paz que proporcione una visión desde las diferentes disciplinas y profesiones e integre los aspectos económicos, culturales, sociales, el ámbito empresarial y las iniciativas de trabajo con comunidades en los procesos de construcción de paz. Los resultados investigativos traslucen los escenarios de futuro para la paz como una forma de estudiar la evolución de la dinámica social y abordar las conflictividades, además de constituir un instrumento que permite analizar la complejidad de la construcción social, como un aporte que orienta a los actores sociales de un proceso de paz en los diferentes cursos de acción y enriquece la práctica social y la retroalimentación de dichas prácticas (Sarti, 2007).

El escenario descrito motiva el estudio realizado, el cual tuvo como objetivo identificar tendencias de futuro para la construcción de paz desde la perspectiva de la educación superior.

\section{Metodología}

Esta investigación se realizó desde un enfoque interpretativo, de tipo documental con el fin de establecer las tendencias de las producciones investigativas sobre construcción de paz y metodologías diseñadas para dicho fin que contribuyan a la proyección de futuro desde la perspectiva de la educación superior. En la fase exploratoria 
se recopiló la información, se consultaron documentos tipo artículo científico, libros, tesis de maestría y doctorado publicados desde el año 2003 hasta el 2019, seleccionados de acuerdo a los criterios de búsqueda establecidos; posteriormente se realizó el proceso de selección y sistematización de la información. A partir de ello se inició la fase de análisis, se procedió a efectuar el análisis de contenido de la información mediante la determinación de categorías y por último la comparación y caracterización de resultados con relación al objeto de estudio.

Los criterios de búsqueda utilizados son: i) Construcción de paz, herramientas y metodologías para la paz, actores para la construcción de paz, escenarios para la paz, iniciativas gubernamentales y sociales para la paz; ii) Modo de publicación: artículos publicados en revistas científicas, tesis de maestría y doctorado iii) lugar e idioma: especialmente en países con historia de conflictos armados y procesos de paz, de acuerdo con el idioma del país en donde se llevó a cabo la investigación. La búsqueda de documentos se realizó en las siguientes bases de datos: Scielo, Dialnet, Redalyc, Scient Diret, Scopus, ProQuest y Google Scholar, en las que fueron seleccionados un total de 80 documentos.

La revisión, selección, organización y sistematización de la información se realizó mediante el estudio exhaustivo de cada documento y el registro sistemático en Excel mediante la identificación previa de: ¿qué se investiga? ¿Dónde se investiga? ¿Cómo se investiga? y ¿Cuáles son los resultados obtenidos? Igualmente, se analizaron los objetivos de las investigaciones relacionadas con el tipo de investigación desarrollada, los enfoques metodológicos, y los aportes más significativos para extraer elementos comunes y relaciones en torno a los procesos de construcción de paz.

\section{Resultados}

Los resultados traslucen los aportes de los estudios analizados y como resultado de la interpretación del contenido se encontraron las siguientes tendencias: i) Elementos y herramientas para la construcción de paz; ii) Iniciativas de construcción de paz; iii) La Educación y el papel de la universidad para la Paz; iv) Proceso sociales, políticos y dialogo para la paz en Colombia; v) Impacto económico y papel de las empresas en el conflicto; vi) Perspectiva-Metodología para la construcción de escenarios futuros. Estas tendencias se presentan teniendo en cuenta los planteamientos de los investigadores y resaltan la validez y representatividad de dichos planteamientos para los procesos de construcción de paz desde la perspectiva de la educación superior, con una discusión de resultados que gira en torno a los aspectos relevantes, aspectos emergentes y aspectos ausentes.

De acuerdo al material de estudio hallado entre el 2004 al 2019, Colombia evidencia un interés significativo en la construcción de material con base al tema de estudio, presenta un $67 \%$ de documentos publicados como se indica en la Gráfico 1. Una de las razones de esta dinámica se basa en la situación del país frente al Conflicto Armado y los intentos por cada gobierno en mitigar y dar solución a este conflicto a través de procesos en iniciativas de paz, en el análisis de posibles soluciones al conflicto a través de negociaciones de paz, los procesos comandados por los expresidentes en el cambio del diseño de contexto interno de Colombia, se convirtió en un importante espacio de investigación entre la relación del gobierno colombiano y la guerrilla insurgente del país FARC-EP (Silva, 2017). Esto explica un interés investigativo por los colombianos, especialmente sobre el análisis de los diferentes programas de desarrollo de paz y laboratorios de paz, para implementar estrategias integrales e identificar las causas directas del conflicto reconociendo actores locales, historias, victimas, culturas, apoyo internacional, interés político, impactos a la sociedad y limitaciones de desarrollo para el país (Ninkov, 2013). 
Gráfico 1

Producción investigativa

por países

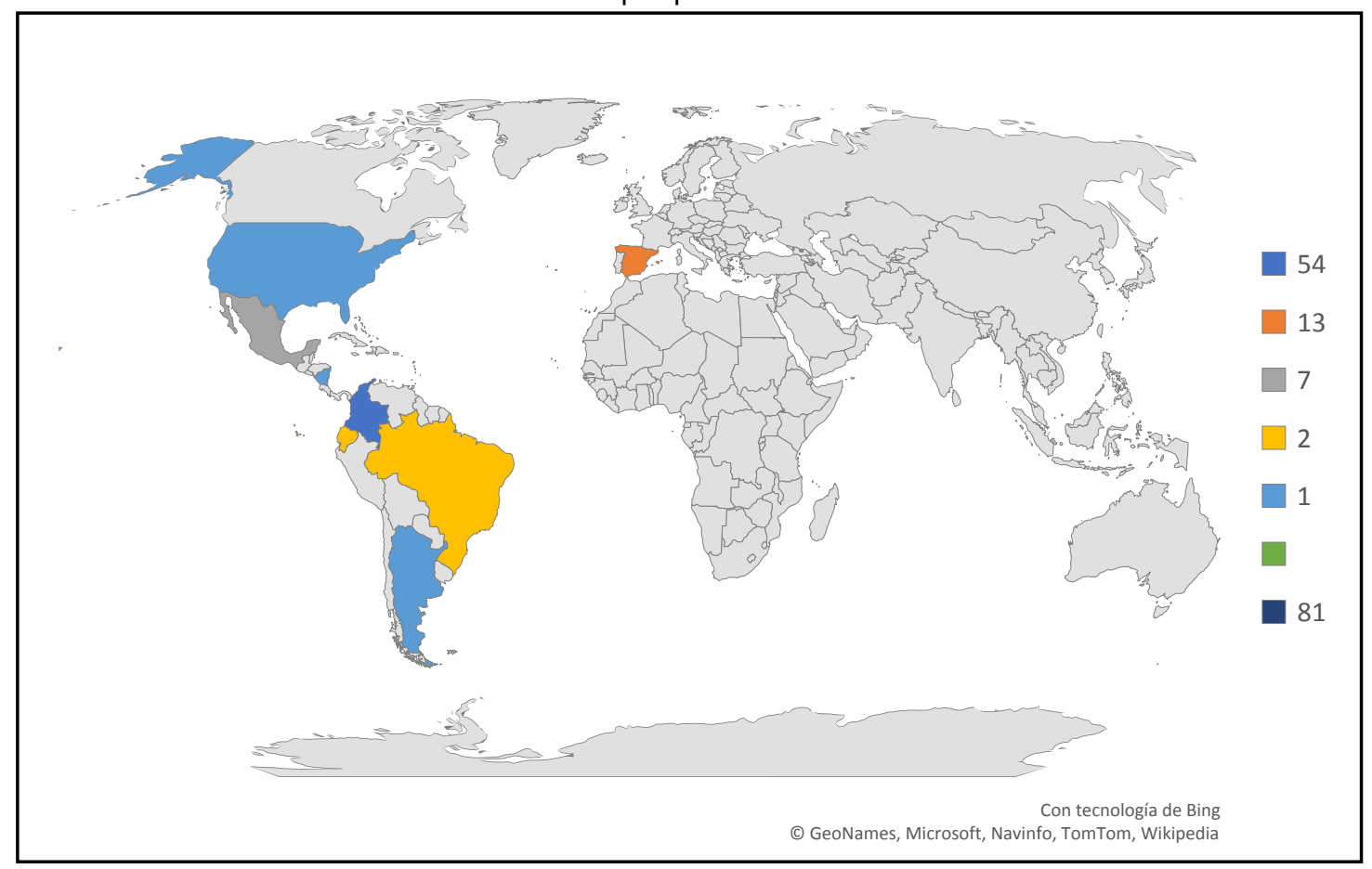

Fuente: Los autores

Otros países han desarrollado investigación en este campo como España 16\%, México 9\%, Brasil 3\%, Ecuador 2\%, Estados Unidos 1\%, Argentina 1\%, Nicaragua 1\%. En los países mencionados se logra establecer que sus investigaciones giran en torno a temáticas que arrojan resultados sobre los impactos del conflicto armado internos y la asociación de violencia política de larga duración, además de ello se reconoce la necesidad de estudiar sobre las raíces del conflicto y las trasformaciones que producen en el tejido social; identificando actores, tiempos, territorios y discursos (Jiménez, 2012).

Con relación a los años en donde se publicaron las diferentes investigaciones, el Grafico No.2 muestra la evolución de las publicaciones y se logra identificar que el año en que se hallaron más documentos sobre la construcción de paz fue en 2014 representando el 19\% del total, en los cuales se habló en su gran mayoría de herramientas y modelos para la construcción de paz, seguido por 2017 con 14\%, en los cuales se puntualiza el papel de la educación para la construcción de paz y la importancia de definir los actores para la construcción de una cultura de paz, al igual que en el 2013 con 10\%, en donde se habló sobre la responsabilidad de la educación y las universidades para la construcción de paz. 
Gráfico 2

Años de producción de los documentos y artículos científicos

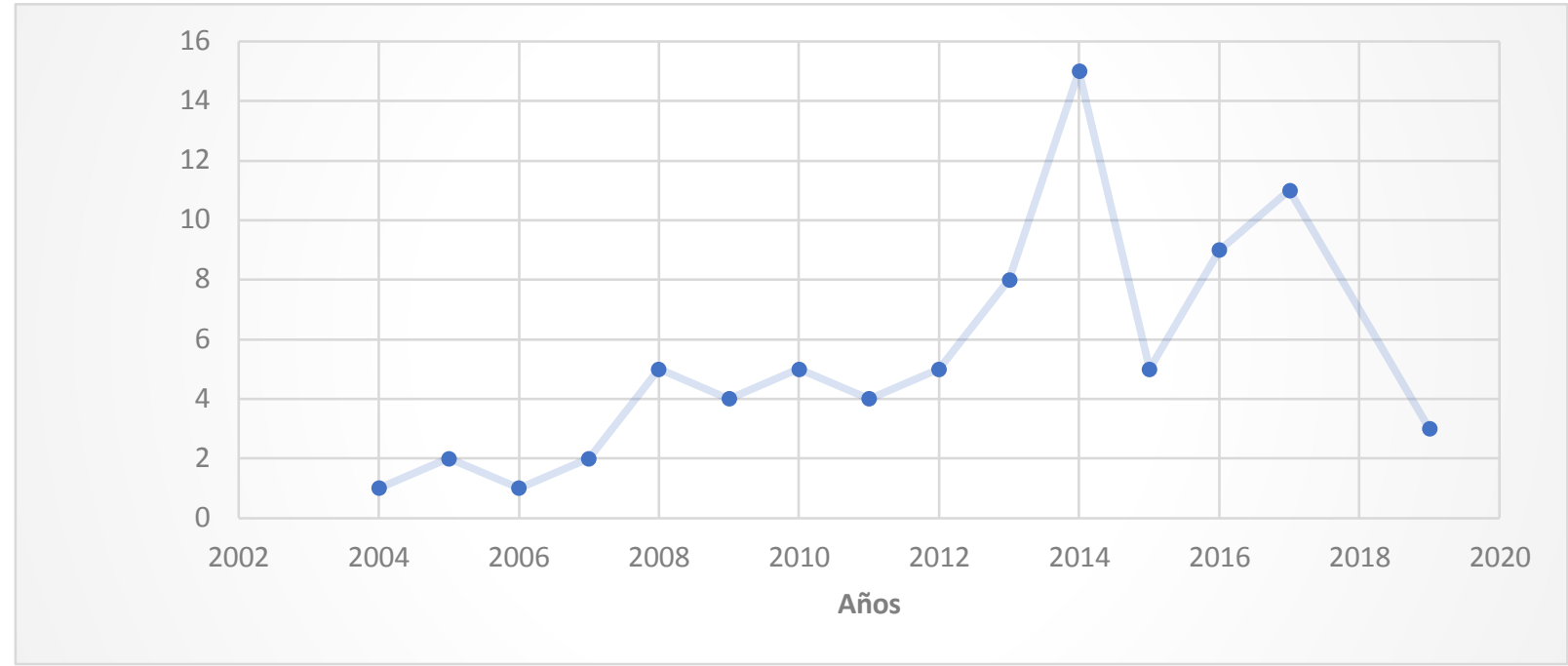

Fuente: Los autores

Así mismo, en el 2016 con 11\%, 2015 con 6\%, 2012 con 6\%, los estudios reflejan reflexiones sobre la educación y el papel de la universidad, también la responsabilidad empresarial para el posconflicto y los gastos del conflicto en Colombia son referentes; en el 2008, representando el 6\%, se habló sobre el posconflicto, el papel de la educación y la evolución de los diálogos de paz; en 2009, con el 5\%, la temática gira en torno a la educación superior y su papel en la construcción social; entre el 2004 y 2007, con el $5 \%$, se habla de los diferentes procesos de diálogos desarrollados por Colombia, su avances retrocesos, problemáticas y beneficios; en el 2015, con 6\%, hace referencia a reflexiones sobre la educación y el papel de la universidad; del año 2017 en adelante se identificaron estudios relacionados con los escenarios de futuro, mecanismos y métodos sobe la construcción de paz, técnicas para la sensibilización de las víctimas del conflicto.

\subsection{Tendencias para la construcción de paz en la educación superior}

Acorde con el análisis realizado, a continuación se presentan las principales tendencias identificadas en las investigaciones, las cuales se presentan de la siguiente forma: i) Elementos y herramientas para la construcción de paz; ii) Iniciativas de construcción de paz; iii) La Educación y el papel de la universidad para la Paz; iv) Procesos sociales, políticos y diálogo para la paz en Colombia; v) Impacto económico y papel de las empresas en el conflicto; vi) Perspectiva-Metodología para la construcción de escenarios futuros.

\subsubsection{Elementos y herramientas para la construcción de paz}

En esta categoría los estudios analizados tienen como propósito reconocer, construir e investigar propuestas de construcción de paz como alternativa para avanzar en la resolución de problemas que aquejan a la sociedad en la búsqueda de paz con la intervención del gobierno, las regiones, la comunidad, el sector privado y la inversión extranjera. Se hallaron estudios entre los años 2007 al 2017 que representan el 15\% del total de los documentos estudiados y producidos mayoritariamente en Colombia, los cuales permitieron identificar la tendencia: Elementos y herramientas para la construcción de paz (Tabla No.1).

Estas investigaciones reflejan los procesos y herramientas empleados para la construcción de justicia y paz, reconociendo la historia del país y los diferentes escenarios políticos para la construcción de paz basados en el perdón y la reconciliación. Algunos autores señalan que las herramientas deben ser diseñadas con y para las comunidades de víctimas, con la inclusión de los diferentes grupos de interés, dentro de los cuales se encuentra 
el gobierno, los medios de comunicación, la inversión extranjera, las comunidades y los grupos al margen de la ley, como alternativa para lograr verdaderos escenarios de construcción de paz.

Bedoya (2014), reconoce que existe una dinámica social y comunitaria interesada en la negociación del gobierno central con la FARC-EP, lo que ha facilitado el diseño de propuestas de cambio y transformación de poblaciones en torno a la paz. También, Amaya (2012) sostiene que las comunidades deben estudiar y reconocer elementos de construcción de justicia, paz y perdón, para así lograr aplicar los enfoques, conceptos y herramientas propuestos para la construcción y la vivencia de la paz, para que trascienda de las propuestas a los medios cotidianos y evitar que sea una paz de "vencederos y vencidos".

Desde esta perspectiva se proyecta la paz de la mano de grandes transformaciones sociales, que permita pasar de los acuerdos y el cese de armas a una paz duradera, para ello es necesario entender las múltiples causas de este fenómeno y el diseño de políticas que propendan por alcanzar una paz duradera, sostenible, plural e incluyente (Salamanca, 2008). Uno de los caminos planteados es la formulación y evaluación de las herramientas y elementos junto a las comunidades y líderes de los procesos de construcción de paz.

Tabla 1

Tendencia elementos para

la construcción de paz

\begin{tabular}{|c|c|c|}
\hline Tendencia & Propósito & Autores \\
\hline $\begin{array}{l}\text { Elementos y herramientas para } \\
\text { la construcción de paz }\end{array}$ & $\begin{array}{l}\text { La inversión extrajera para dicho fin } \\
\text { y la unión entre sectores públicos y } \\
\text { privados, siempre orientados a } \\
\text { resolver problemas que aquejan a la } \\
\text { sociedad en la búsqueda de paz. }\end{array}$ & $\begin{array}{l}\text { Acosta, Montoya, Gallego., \& Garcés (2017); } \\
\text { Bernardelli (2014); González (2007); Marín, } \\
\text { García Gonzales, \& Buriticá (2013); Rettberg, } \\
\text { et al. (2017); Arroyo y Alvarado (2016); Mur } \\
\text { y Contreras (2019). }\end{array}$ \\
\hline
\end{tabular}

Fuente: Los autores

\subsubsection{Iniciativas en la construcción de paz}

En las investigaciones se establecen propuestas y modelos de construcción de paz desde diferentes perspectivas, de igual manera se señala la necesidad del trabajar con las comunidades a través de laboratorios de paz, el diseño de metodologías para formular iniciativas en la construcción de paz. Esta dimensión estudia el 16\% del total de las investigaciones consultadas, publicados entre los años 2005 al 2017, y expresa la importante de los enfoques de construcción de paz se funden en estudios sistemáticos a mediano y largo plazo, aspectos que llevaron a identificar la tendencia: Iniciativas en la construcción de paz (Tabla 2).

Lo expuesto afianza la construcción de escenarios factibles y aplicables, además deben estar acompañados por actores externos que posan un alto nivel de conocimiento, comprometidos hacia alcanzar la sostenibilidad del proceso (Katz, 2011). Además, lo planteado por Álvarez- (2017) reafirma que una de las mejores estrategias para la construcción de escenarios de paz debe estar fundamentada en la educación para la paz; la formación se debe enfocar en la construcción de proyectos pedagógicos, estrategias de enseñanza y aprendizaje de valores, hábitos y competencias para la paz, con el propósito de minimizar los impactos de la violencia en todos los ámbitos, desde conflictos políticos, sociales e interpersonales y de buscar la trasformación del conflicto a formas legales y negociables.

Igualmente, Ávila (2013) justifica la razón por la cual el Estado y la sociedad debe asumir una actitud efectiva frente a los problemas y las violaciones de los derechos de las poblaciones víctimas del conflicto, por ello se deben construir mecanismos y escenarios que permitan mejorar la convivencia hacia el trato digno en las distintas poblaciones, además suscitar movimientos sociales que luchan por los derechos humanos. 
Tabla 2

Tendencia escenarios de paz

\begin{tabular}{|c|c|c|}
\hline Tendencia & Propósito & Autores \\
\hline $\begin{array}{c}\text { Iniciativas en la construcción } \\
\text { de paz. }\end{array}$ & $\begin{array}{l}\text { El papel de gobierno, la comunidad y } \\
\text { los actores del conflicto, para formar } \\
\text { iniciativas de paz en la búsqueda de } \\
\text { prácticas de construcción de paz. }\end{array}$ & $\begin{array}{l}\text { Baena (2009); Rettberg (2013); Álvarez (2017); } \\
\text { Balbi (2010); Beil (2006); Barreto (2016); } \\
\text { Rosanía (2014); Parra (2014); Barbero (2006); } \\
\text { Muslera (2016); Smith (2017). }\end{array}$ \\
\hline
\end{tabular}

Fuente: Los autores

\subsubsection{La educación y el papel de la universidad para la paz}

Esta categoría analiza el papel de la educación como cimiento para el desarrollo social y destaca el papel del sistema educativo, fundamentalmente en Colombia, como elemento indispensable en la transición entre una cultura de la violencia hacia una cultura de paz. Al desarrollar esta dimensión se hallaron documentos de carácter investigativo que representan el $23 \%$ del total de estudios, con origen de México y Colombia que permitieron identificar la tendencia: La educación y el papel de la universidad para la paz (Tabla 4). Los aportes de la educación para la construcción de paz se basan en el diseño de paradigmas de bienestar y buen vivir para generar fortalecimiento institucional y regional como núcleos de la formación humanística; considerando que todo profesional debe tener capacidades humanísticas y sociales para la construcción de paz (Padilla, 2017).

Se destaca lo expresado por Salguero y Seva (2005), quienes argumentan la necesidad de una cultura de paz que exige cambiar de un modelo de educación institucionalizada a un modelo de sociedad educativa, el cual debe capacitar en derechos humanos para la paz, así como en derechos humanos sostenibles. Para la construcción de escenarios de paz las universidades se constituyen en líderes de la formación humanística, el desafío está en reconocer a dichas instituciones como protagonistas del proceso, para ello los docentes y estudiantes requieren de programas curriculares que mejoren los procesos de enseñanzas para fortalecer y aplicar mejoras a comunidades y organizaciones sociales en materia de construcción de paz (Valenzuela, Guillén, Medina, \& Rodríguez, 2017).

Además, Peña (2015), señala la necesidad de crear:

“Programas de educación superior en lo que concierne a la desmovilización y las víctimas del conflicto armado, ya que los pocos que existen están dirigidos a generar conciencia con respecto a la convivencia y la cultura de paz; la educación superior debe crear unos procesos diferenciales de formación hacia la paz y los procesos de reintegración de excombatientes".

Es importante mencionar que los semilleros de investigación en las universidades se convierten en un espacio estratégico de dialogo para generar estrategias de mejoramiento y solución a problemas de violencia y construcción de paz (Alvarado y Hernández, 2015). En consecuencia, la educación para la paz requiere de un proceso permanente de seguimiento que transcienda de un periodo gubernamental que garantice la correcta interpretación y aplicación de estrategias y acciones con base a rutas metodológicas de paz hacia comunidades en el largo plazo (Carrillo, 2014). 
Tabla 3

Tendencia educación para la paz

\begin{tabular}{lll}
\hline \multicolumn{1}{c}{ Tendencia } & \multicolumn{1}{c}{ Propósito } & \multicolumn{1}{c}{ Autores } \\
\hline La educación y el papel de & Análisis de propuestas educativas para la paz & Cruz (2008); Fajardo (2016); Franco, \\
la universidad para la paz & con la articulación del ámbito social y & Bautista, \& Carmona (2009); Pérez \\
& educativo, que contribuyan a la construcción de & (2015); Sánchez (2010); Infante (2014); \\
& nuevas prácticas para el manejo del conflicto y & Barón, Pedraza, y Hendez (2009); \\
& el postconflicto, indicando el papel de los & Baquero y Landínez (2014); Clavijo \\
& diferentes actores que participan en la & (2010); Machado (2011); Rivero \\
& construcción de paz. & (2017). \\
\hline
\end{tabular}

Fuente: Los autores

\subsubsection{Proceso sociales, políticos y diálogos para la paz en Colombia}

Las investigaciones relacionadas en esta categoría exponen los diálogos de paz desarrollados en Colombia, como un proceso dinámico construido con una estructura de oportunidades para la formulación de alternativas en el desarrollo de la reconciliación y la transición hacia la democracia. En el estudio de esta tendencia es el resultado del 13\% de las investigaciones publicadas entre los años 2003 al 2019, tienen como propósito describir y evaluar las posiciones del estado, el sector público y privado frente a los problemas del postconflicto y negociación de la paz, se identifica la tendencia: Proceso sociales, políticos y diálogos para la paz en Colombia (Tabla 4.).

Con relación a esta tendencia Olave (2013), analiza los discursos de paz del Estado con la FARC-EP, en los cuales se reconoce a la paz como una oportunidad histórica que representa una gran responsabilidad y con riesgos significativos que requiere del consenso institucional y social; sin embargo, se reconoce que cada actor efectúa acciones de acuerdo a su interés político y con poca relación al contexto social del país. Los diálogos de paz que se han llevado a cabo en la historia de Colombia se han cimentado en negociaciones de agendas restrictivas, las cuales se limitan a la entrega de armas, desmovilización y reinserción, sin lograr negociar una paz estable y duradera (Loaiza, 2012).

Además, la paz sostenible implica el proceso de transformación representado por el paso de una etapa y enfrentamiento a una negación y relaciones pacíficas, de una condición de extrema vulnerabilidad y dependencia a una autosuficiencia y bienestar, y de un espiral de violencia y destrucción a un espiral de paz y desarrollo (Barreto, 2016). No obstante, mientras los actores armados continúen irrespetando las normas de la guerra, los derechos de amparo y el Estado siga soslayando su compromiso constitucional para garantizar los derechos humanos, no se puede pensar en una salida efectiva al conflicto armado en Colombia (Pérez, 2011).

Tabla 4

Tendencia Procesos Sociales y Políticos

\begin{tabular}{lll}
\hline \multicolumn{1}{c}{ Tendencia } & \multicolumn{1}{c}{ Propósito de los estudios } & \multicolumn{1}{c}{ Autores } \\
\hline Proceso sociales, políticos y & Diálogo y negociación del proceso de & Herrera (2009); Rey (2010); Arévalo (2014). \\
diálogos para la paz en & paz en Colombia: oportunidades y & PNUD (2019); Jiménez (2012); Loaiza (2012), \\
Colombia & limitaciones & Olave (2013). \\
\end{tabular}

Fuente: Los autores

\subsubsection{Impacto económico y papel de las empresas en el conflicto}

En esta categoría de estudio se identifica como propósito determinar la estructura de costos del conflicto armado para comprender la responsabilidad social empresarial en la construcción de paz como insumo para elaborar un análisis prospectivo del postconflicto frente a la firma de los acuerdos de paz. Esta tendencia corresponde al 10\% del total de los estudios analizados, con los cuales se identificó: Impacto económico y papel de las empresas en el conflicto (Tabla 5). 
El sector empresarial debe ser más consciente de la responsabilidad social como uno de los compromisos que deben asumir frente a los nuevos tratados de paz en los que se destacan aspectos como la reintegración de excombatientes a la vida laboral para mejorar el contexto y la interacción entre empresa-sociedad, aspectos que inciden en la calidad de vida de una sociedad (Villamarin, 2016). En este sentido, la responsabilidad social empresarial favorece los procesos de construcción de paz, que demandan mayor participación del sector empresarial tanto en el contexto formativo como en el laboral (Santamaría y Grueso, 2019).

El conflicto armado afecta a diversas comunidades, es por ello que las empresas socialmente responsables deben realizar una contribución a la paz, para ello es necesario adoptar herramientas implementadas por diversas iniciativas globales, dentro de las cuales se destaca la vigilancia a sus propias actividades empresariales para que no fomenten directamente en las zonas de conflicto los intereses económicos, políticos y culturales desconociendo las necesidades particulares de las poblaciones (Vargas, 2014).

Tabla 5

Tendencia Impacto económico

\begin{tabular}{lll}
\hline \multicolumn{1}{c}{ Tendencia } & \multicolumn{1}{c}{ Propósito } & \multicolumn{1}{c}{ Autores } \\
\hline Impacto económico y papel de & Responsabilidad social en la & Álvarez y Rettberg (2008); Peña (2014); Tercero \\
las empresas en el conflicto. & construcción de Paz & (2016); Prieto (2017), Santamaría \& Grueso \\
& & (2019).
\end{tabular}

Fuente: Los autores

\subsubsection{Perspectiva metodológica para la construcción de escenarios}

Las investigaciones analizadas en esta categoría tienen como intención de describir la necesidad de la prospectiva y sus herramientas metodológicas y estratégicas a partir de la construcción de escenarios para evaluar y explicar las causas de la violencia, así como también la identificación de estrategias de fortalecimiento y desarrollo de los programas curriculares en el marco de los posibles escenarios futuros. El análisis se realizó entre los años 2009 a 2017, representando el $23 \%$ de los documentos, que detallan aspectos metodológicos, iniciativas y visiones para la construcción de paz con el aporte de comunidades y trabajadores sociales, que permiten identificar la tendencia: Perspectiva metodológica para la construcción de escenarios (Tabla 6).

En las investigaciones se analizan el término prospectiva, enfocado en describir la necesidad de la prospectiva en el ordenamiento territorial, los encuadres metodológicos, las fases de acción y las opciones estratégicas a partir de la construcción de escenarios, para que a partir de ello se logre entender, evaluar y explicar las causas de violencia. También se relaciona informes sobre conflicto, derechos humanos y construcción de paz y los escenarios de riesgos como herramienta metodológica prospectivas que permitan la creación de capacidades en las Instituciones de Educación Superior a partir de la identificación de estrategias de fortalecimiento y desarrollo de los programas curriculares en el marco de los posibles escenarios futuros.

Los escenarios para la construcción de paz delimitan los caminos que permiten tener una visión del futuro de dicho conflicto (Ciriza, 2014), revelan los escenarios de riesgos como instrumento de la prospectiva que permite la creación de capacidades en las Instituciones de Educación Superior a partir de la formulación de estrategias de fortalecimiento y desarrollo de los programas curriculares en el marco de los posibles escenarios futuros. Como lo expresan Argueta, Agudelo \& Cardona (2016), la planeación de escenarios permite reconocer las brechas y oportunidades para el objeto de estudio; facilita el diseño de escenarios alternativos y permite iniciar la fase estratégica de la planificación en la cual se define las rutas para alcanzar el escenario apuesta diseñado (Castillo, 2012).

Los escenarios prospectivos pretenden crear realidades futuras, como medio para dar solución a problemas específicos, en este caso la terminación de la guerra, se deben generar estrategias en cada uno de los sectores 
económicos de la sociedad para garantizar una política equilibrada como mecanismo de desarrollo de los países, conociendo que el escenario del posconflicto presenta un gran reto para la consolidación de una paz estable y duradera (Sánchez, 2013).

Tabla 6

Tendencia metodologías escenarios de futuro

\begin{tabular}{lll}
\multicolumn{1}{c}{ Tendencia } & \multicolumn{1}{c}{ Propósito } & Autores \\
\hline Perspectiva & Escenarios de futuro y aporte al & Godet y Vásquez (2011); Valencia, Benjumea, \\
metodológica para la & proceso de construcción de paz & Peláez, \& Orozco (2013); Montes (2014); Muñoz \\
construcción de & & (2014); Navarro, Royo, Urgell, Urrutia \& Villelas \\
escenarios & (2016); Peláez, Álvarez, Palacio \& Mazo (2017). \\
\hline
\end{tabular}

Fuente: Los autores

\subsection{Propósitos y enfoques de las investigaciones objeto del estudio}

En este apartado de la investigación se da respuesta al interrogante: ¿para qué se investiga?, teniendo en cuenta que los objetivos investigativos presentan una estrecha relación entre la metodología y los resultados obtenidos e inciden en el tipo de investigación llevada a cabo. Al respecto, Salamanca (2008) proyecta identificar y desarrollar posibilidades de asociación entre regiones víctimas del conflicto para lograr mayor compresión de las problemáticas regionales y sectoriales como estrategia de cooperación. Asimismo, Franco, Bautista, \& Carmona (2009) plantean analizar la estructura, el símbolo y la cultura detrás de los conflictos armados en un país para determinar los principios de formación en las comunidades sobre el tema de mitigación y efectos de los conflictos.

En los estudios exploratorio identificados se emplearon verbos como: identificar, evidenciar, revisar y principalmente examinar lineamientos para los programas o acciones de construcción de paz, especialmente se señalan la importancia de trabajar con las comunidades y para las comunidades. Al respecto, Rodríguez (2013) propone identificar elementos de construcción de paz e incorporar programas colectivos de música en víctimas del conflicto armado en Colombia; igualmente, Abrego (2009) contextualiza proyectos sobre el papel de las universidades en la construcción de paz y la necesidad de conocer el interés de la comunidad internacional en la promoción de construcción de paz en Colombia desde la perspectiva social y privada (Rettberg, 2010).

Los objetivos de las investigaciones de tipo descriptivo halladas en el estudio buscan caracterizar, describir, estudiar, determinar, espacios de construcción de paz de origen local y no gubernamental para la construcción de paz y transformación de conflictos (Bernardelli, 2014). Las comparaciones de los documentos hallados sobre el tema de estudio presentan un mismo problema en común como base de investigación, la manera en cómo es abordado y tratado depende del fin de cada autor y las necesidades de comunidades, actores, culturas y espacios geográficos.

Durante el análisis de los estudios hallados se pretende dar respuesta a la pregunta ¿cómo se está investigando? Y de acuerdo a los objetivos de los estudios, las investigaciones más usadas son de tipo descriptivo y exploratorio, primando estudios documentales, históricos, experimentales e interactivos-observatorios. Los paradigmas metodológicos son de tipo cualitativo, cuantitativo y mixto -cuali-cuantitativo-; los instrumentos de mayor uso son: trabajo de campo, estudio de casos, análisis documental, entrevistas, encuestas, trabajo con comunidades.

El enfoque más utilizado es el cualitativo (82\%), predominan investigaciones relacionadas con la construcción de paz mediante el análisis descriptivo, análisis de informes de opinión, dialogo reflexivos, marcos teóricos y estados del arte que estudian las herramientas, mecanismos y actividades para la construcción de paz, además se indaga la evolución de los acuerdos de paz en Colombia y el papel de los organismos involucrados en el proceso. El 
enfoque cuantitativo es un complemento en estos tipos de investigación, prácticamente se usó para análisis de datos, encuestas y mapeos de zonas estudiadas (18\%).

La construcción de escenarios se mencionó como una metodología acompañada de herramientas como Brainstorming o lluvias de ideas, matriz de impactos cruzados, matriz decisional, empleada para el desarrollo de investigaciones de carácter inductivo, analizando realidades sociales a partir de la exploración y la descripción, para luego generar conocimiento a partir de conceptos teóricos de los posibles escenarios futuros del objeto de estudio.

\subsection{Discusión}

En las tendencias de publicaciones se observa el tópico investigativo con énfasis en Colombia frente al tema de estudio, lo cual puede ser generado por la violencia que ha marcado la historia de este país. Las temáticas más abordadas se basan en la cultura para la paz, la formulación y aplicación de tratados, la negociación, la participación de los diferentes actores, la educación para la paz, la construcción de escenarios y el trabajo con comunidades víctimas.

La necesidad de aplicar justicia, restituir los derechos de las víctimas y consolidar los equilibrios dinámicos superiores como instancias sucesivas de construcción de paz llevan a analizar el proceso de paz desde sus oportunidades históricas, los diferentes riesgos y responsabilidades, también el consenso y la excepción bélica. Es importante señalar que mientras los actores armados continúen irrespetando las normas de la guerra, los derechos que amparan y salvaguardan la existencia y bienestar de la sociedad civil y el Estado siga soslayando su compromiso constitucional de garantizar el cumplimiento de los derechos humanos, especialmente aquellos orientados hacia los desplazados, incrementará cada día el número de víctimas de los actores armados y los sectores económicos, sociales y políticos excluirán a estas poblaciones negándole la garantía socioeconómica que les permiten tener una vida digna, en consecuencia difícilmente se podrá pensar en una salida efectiva al conflicto armado, por ejemplo en Colombia.

Desde la perspectiva metodológica, los escenarios constituyen un instrumento de planificación que contribuye a la construcción de la propuesta de escenarios de futuro para la paz. Los escenarios exploratorios, como fase inicial de la metodología permiten describir la posible evolución futura de los acontecimientos actuales, para que partir de dicha información se logre la construcción de escenarios deseados de acuerdo al contexto problemático, es decir se busca aprender de los errores antes de cometerlos, más que describir una acción o una situación los escenarios ayudan a moldear aspectos del tema investigado, por ello algunos autores hablan de los escenarios prospectivos y los cognitivos. Mientras los primeros se encargan de identificar escenarios probables, los segundos buscan ampliar el conocimiento como aporte al ejercicio de planeación.

Como temas viables para el desarrollo de nuevas investigaciones, se resalta el interés de indagar, en Colombia, sobre el papel del Estado en el cumplimiento de los acuerdos pactados con las FARC-EP, el seguimiento a procesos de paz y cultura de paz en comunidades afectadas por el conflicto, así como la efectividad de la aplicación de escenarios como metodologías para prevenir errores en la construcción de mecanismo para la paz; es clave señalar la incidencia sobre el papel de la universidad como entes formadores de profesionales hacia la paz y las empresas como entidades colaboradoras en procesos de reintegración de víctimas y excombatientes. A continuación, se presentan los aspectos considerados como relevantes, emergentes y ausentes en el presente análisis:

Lo relevante: En el desarrollo de los procesos de construcción de paz se puede alcanzar una comprensión de la necesidad de generar técnicas y herramientas para la construcción de justicia, paz y reconciliación, dicha necesidad surge a partir de la caracterización y el mapeo de iniciativas de paz y la historia de los diferentes escenarios políticos que han surgido para llevar a cabo una paz basada en el perdón y la reparación. En este 
sentido Villa (2016) afirma que también la impunidad dificulta procesos de perdón. Al respecto son relevantes los trabajos que promueven diferentes iniciativas de paz, como diálogos entre los actores sociales y su conocimiento sobre capacidades locales de paz Katz (2011); Rettberg (2010); Bernardelli (2014). Además de la importancia de un modelo de comunicación para el desarrollo de paz que genere las condiciones el desarrollo humano y la paz (Bedoya, 2014).

Es decir, la construcción de paz se fundamenta en un cambio social como elemento indispensable para una transformación sostenible del conflicto, este proceso debe ir acompañado de una inversión que busque mejorar la calidad de vida de la población, para generar mayor confianza en los procesos. Un modelo Paz justa abarca tres modos básicos de activismo en la consolidación de la paz: la abogacía organizada para la paz y la reconciliación, la promoción del diálogo entre las partes en conflicto y la acción directa no violenta en contra de la represión violenta y el uso injustificado de la fuerza (Smith Jr,2017).

Lo emergente: Según las investigaciones modernas en las áreas sociales, se despierta el interés por conocer y comprender el futuro, en esta ruta los diferentes aportes han consolidado los trabajos con las metodologías de escenarios como un punto de partida idónea para reconocer y analizar aquellas fuerzas que determina y moldean un futuro frente a un hecho concreto, para que a partir de ello se logre la construcción de estrategias que se puede aplicar a los posibles desafíos que se presenten. Estas tendencias están en las investigaciones de Rettberg (2013), Mateos (2012) y Ávila (2013). Por ello los escenarios son herramientas que permiten crear un lenguaje común entre la realidad y un imaginario de como dicha realidad se transformaran en un futuro (Beil, 2006; Balbi, 2010).

Los escenarios desde la prospectiva es una herramienta empleada en la planificación, ya que desde la perspectiva se logra construir escenarios, desde un inicio se crean escenarios exploratorios, los cuales permiten describir la posible evolución futura de los acontecimientos actuales, para que partir de dicha información se logre la construcción de escenarios deseados de acuerdo a la problemática planteada (Álvarez ,2017). Asimismo, se busca aprender de los errores antes de cometerlos, más que describir una acción o una situación los escenarios ayudan a moldear aspectos del tema investigado, por ello algunos autores hablan de los escenarios prospectivos y los cognitivos (Baena, 2009).

Estas metodologías emergentes como los escenarios son análisis que además de establecer posibles futuros en la construcción del proceso de paz en las zonas de conflicto, también permiten organizar la dinámica que deben desempeñar los actores responsables para lograr un futuro deseado (Blois y Osuza, 2008). Al respecto, el término escenario ha sido usado en dos sentidos: el primero, describir una foto instantánea en el tiempo o de condiciones de variables importantes en algún tiempo particular en el futuro; el segundo, describir una historia futura esto es, la evolución desde las presentes condiciones a uno o varios futuros (Balbi,2004).

Lo ausente: En el desarrollo de las investigaciones estudiadas se refleja una amplia identificación de la necesidad de dar punto final a la problemática del Conflicto Armado, pero no se tiene diseños ni estructuras curriculares que permitan un cambio en los imaginarios de la población donde se encuentran las nuevas generaciones que terminan haciendo una adaptación del vivir en medio del conflicto. Además, los programas o lineamientos existentes adolecen de acciones puntuales de ciudadanía enmarcadas en la transformación de los conflictos a través de una cultura de la negociación y de estrategias de reconciliación, sin embargo, algunos lineamientos de política pública hacen alusión a la continuidad del proceso de educación inclusiva y sobre el cual el enfoque de derechos es fundamental (Carrillo y Martinez, 2015). 


\section{Conclusiones}

Resultado del análisis se identificaron seis tendencias de futuro en las que se destaca el papel de la Educación Superior en los procesos de construcción de paz, en la formulación de inciativas y herramientas que aporten a la construcción de los diferentes procesos sociales y políticos que impacten los ecosistemas de transformación productiva en el desarrollo de escenarios que contribuyan al fortalecimiento de capacidades institucionales, integrando la triada: Educación, Empresa, Estado, enfocados en los retos y desafíos que se presenten en el futuro.

Los resultados más significativos con relación a la construcción de paz, sus metodologías, políticas y procesos empleadas para dicho fin evidencian la necesidad de generar procesos y herramientas para la construcción de justicia, paz y reconciliación, esta necesidad surge a partir de la caracterización y el mapeo de iniciativas de paz en los territorios y la historia de los diferentes escenarios políticos que han surgido para llevar a cabo una paz basada en el perdón y la reparación. Los diversos instrumentos diseñados a partir del trabajo con comunidades víctimas y la sistematización sobre pensamientos de justicia ancestral se fundan en propuestas de modelos de comunicación para la paz y las perspectivas de la sociedad civil integrada con el sector privado.

La construcción de paz se fundamenta en un cambio social como elemento indispensable para una transformación sostenible del conflicto, este proceso debe ir acompañado de una inversión que busque mejorar la calidad de vida de la población para generar mayor confianza en los procesos. Un modelo de Paz justa, abarca tres modos básicos de activismo en la consolidación de la paz: la abogacía organizada para la paz y la reconciliación, la promoción del diálogo entre las partes en conflicto y la acción directa no violenta en contra de la represión y el uso injustificado de la fuerza.

Como punto de congruencia las investigaciones consultadas reconocen que la paz no se construye mediante firmas de tratados, sino en la medida que se logre una voluntad colectiva apoyada en la educación, para que así se logre una buena voluntad y convivencia pacífica que no sea limitada por el cumplimiento de puntos negociados. Ahora el reto está en los educadores y formadores, en los universitarios e intelectuales, de contribuir desde diferentes espacios para transformar sentimientos, posturas y valores que han alimentado el círculo de violencia, exclusión y odio.

Para lograr metodologías de escenarios prospectivos es necesario una nueva política de fortalecimiento de las capacidades institucionales, que refuerce el componente educativo, la empresa, el estado y la política pública, creando lineamientos de estrategias institucionales enfocadas a los retos y desafíos que se presente en análisis futuros.

\section{Referencias bibliográficas}

Acosta Hoyos, H., Montoya Gómez, J. D., Gallego López, C. E., \& Garcés Caicedo, M. D. S. (2017). Construcción de cultura de paz, como una posibilidad para la resignificación del desarraigo en víctimas del desplazamiento que habitan en el proyecto "Valle del Ortigal", Popayán-Cauca. Recuperado de http://ridum.umanizales.edu.co:8080/xmlui/handle/6789/3106

Abrego Franco, M. G. (2009). Propuesta de educación y cultura de paz para la ciudad de Puebla (México). Recuperado de https://digibug.ugr.es/bitstream/handle/10481/2161/17821496.pdf?sequence=1\&isAllowed=y

Alvarado, M. L., y Hernández, S. A. (2015). La universidad frente a la construcción de paz: claves para una convivencia posible y duradera. Revista de la Universidad de La Salle, (67), 95-105. Recuperado de https://revistas.lasalle.edu.co/index.php/ls/article/view/3626 
Álvarez, A. (2017). Acuerdos y construcción de paz en Colombia: retos a la gobernabilidad y la cultura de paz. PROSPECTIVA. Revista de Trabajo Social e Intervención Social, (24), 13-45. Recuperado de file://D:/Datos\%20Usuario/Downloads/5872-157-15466-1-10-20171103.pdf

Álvarez, S., y Rettberg, A. (2008). CUANTIFICANDO LOS EFEC TOS ECONÓMICOS DEL CONFLICTO: una exploración de los costos y los estudios sobre los costos del conflicto armado colombiano. Colombia internacional, (67), 14-37. Recuperado de https://revistas.uniandes.edu.co/doi/abs/10.7440/colombiaint67.2008.01.

Álvarez, A. A. (2017). Acuerdos y construcción de paz en Colombia: retos a la gobernabilidad y la cultura de paz. PROSPECTIVA. Revista de Trabajo Social e Intervención Social, (24), 13-45. Recuperado de file://D:/Downloads/5872-157-15466-1-10-20171103.pdf

Amaya, J. (2012). Legado y contribución del pensamiento carare a la construcción de paz en Colombia (Master's thesis, Facultad de Ciencias Políticas y Relaciones Internacionales). Recuperado de https://repository.javeriana.edu.co/handle/10554/2365

Arroyo Ortega, A., y Alvarado Salgado, S. V. (2016). Conocimiento en colabor: Reflexiones y posibilidades para la construcción de paz. Recuperado de https://dspace.ups.edu.ec/handle/123456789/14093

Argueta, C., Agudelo, I., \& Cardona, O. (2016). Planeación por escenarios: un caso de estudio en una empresa de consultoría logística en Colombia. Estudios Gerenciales, 32(138), 96-107. Recuperado de https://www.sciencedirect.com/science/article/pii/S012359231600005X

Arévalo, J. (2014). Construcción de paz y un nuevo modelo de construcción de estado: Una lectura de los dos primeros acuerdos de La Habana (Peacebuilding and a New Model of State-Building: A Reading of the First Two Agreements of Havana). Revista de economía institucional 16(30). Recuperado de https://papers.ssrn.com/sol3/papers.cfm?abstract_id=2455866

Ávila, M. (2013). La ruta pacifica de las mujeres y su aporte en la construcción de paz en Colombia estudio de caso (Bachelor's thesis, Facultad de Ciencias Políticas y Relaciones Internacionales). Recuperado de https://repository.javeriana.edu.co/handle/10554/15220.

Baquero Rodríguez, M. J., y Landínez Ariza, P. A. (2014). Educación, paz y posconflicto: oportunidades desde la educación superior. Revista Universidad de La Salle (65), 115-134. Recuperado de http://revistas.lasalle.edu.co/index.php/Is/article/view/3357

Baena, G. (2009). Prospectiva por qué y para qué: la historia que muchos no quieren leer. Estudios políticos (México), (17), 109-127. Recuperado de http://www.revistas.unam.mx/index.php/rep/article/view/23786

Balbi, E.R. (2004) Escenarios por The Futures Group. (versión en español) traducción corresponde a la Sección № 08 de la publicación “Futures Research Methodology, Versión 1.0", de Jerome C. Glenn, Editor, publicada por el Millennium Project del American Council for the United Nations University, Washington, USA, 1999. ISBN: 0-9657362-2-9, recuperado de http://saludpublicavirtual.udea.edu.co/cvsp/politicaspublicas/futures_group_escenarios.pdf

Balbi, E. (2010). Manual Básico del Método Oficial de Prospectiva de la Red EyE (Escenarios y Estrategia) en América Latina. Argentina. 77pp. Disponible en: http://190.104, 117, 2014.

Barbero, A. (2006). Construyendo paz en medio de la guerra: Colombia. Barcelona: Universidad Autónoma de Barcelona.

Barón, M., Pedraza, J., \& Hendez, I. R. (2008). Los retos de la responsabilidad social universitaria: construyendo paz desde la universidad. Revista Educación Superior y Sociedad (ESS) ISSN: 0798-1228, 13(2), 6390.Recuperado de https://www.iesalc.unesco.org/ess/index.php/ess3/issue/view/37/38. 
Barreto, M. (2016). Laboratorio de paz en territorios de violencia(s) ¿abriendo caminos para la paz positivas en Colombia? Universidad de Bogotá Jorge Tadeo Lozano- Facultad de Ciencias Sociales. ISBN. 978-958-725192-0. Recuperado de https://www.utadeo.edu.co/sites/tadeo/files/node/publication/field_attached_file/pdf_laboratorios_de_paz_web-16.pdf

Bedoya, T, G. (2014). Construcción de un modelo de comunicación para el desarrollo y la paz en el oriente antioqueño (Colombia) (Doctoral dissertation, Universidad de Granada). Recuperado de https://dialnet.unirioja.es/servlet/tesis?codigo=57411.

Beil, A. (2006). Destino Colombia: Crónica y evaluación de un ejercicio de participación de líderes de la sociedad civil en el diseño de escenarios futuros. Universidad de los Andes, Fac. de Ciencias Sociales, Depto de Ciencia Política, CESO, Ediciones Uniandes. Recuperado de https://repositorio.uniandes.edu.co/handle/1992/8019?show=full.

Bernardelli, L. (2014). Aproximaciones a iniciativas locales de construcción de paz en Colombia (Doctoral dissertation, Bogotá: Universidad Nacional de Colombia, 2014). Recuperado de http://www.bivipas.unal.edu.co/handle/10720/682.

Blois, H. D., \& Souza, J. C. (2008). Cenários prospectivos e a dinâmica de sistemas: proposta de um modelo para o setor calçadista. RAE-Revista de Administração de Empresas, 48(3), 35-45. Doi: 10.1590. Recuperado de http://bibliotecadigital.fgv.br/ojs/index.php/rae/article/view/36655.

Carrillo, J. (2014). La Educación superior como vector de paz en Colombia - Lineamientos. Ministerio de Educación Nacional (En el marco del contrato 050 de 2014). Recuperado de http://www.universidad.edu.co/images/cmlopera/descargables/lineamientos_educacioon_inclusiva.pdf.

Carrillo, J,. y Martínez, P.(2015). La educación superior como vector de paz en Colombia". Pensamiento universitario $N^{\circ} 26$, ASCUN, 2015, p. 20-26.Recuperado de https://www.academia.edu/10813597/CARRILLO_Juan_Felipe_and_MART\%C3\%8DNEZ_Patricia_La_educa ci\%C3\%B3n_superior_como_vector_de_paz_en_Colombia_en_Pensamiento_universitario_n_26_ASCUN_ 2015_p_20_26

Castillo, J. (2012). Generación de escenarios prospectivos que sustenten la planificación de la seguridad del estado hasta el año 2015 (Master's thesis, Quito, Ecuador). https://repositorio.iaen.edu.ec/xmlui/handle/24000/3691.

Cerdas, E. (2015). Desafíos de la educación para la paz hacia la construcción de una cultura de paz. Revista Electrónica Educare, 19(2), 135-154. Recuperado de https://dialnet.unirioja.es/servlet/articulo?codigo=5053321

Ciriza, J. (2014). Escenarios prospectivos de resolución de conflicto. Bases para el diseño de escenarios futuros de resolución del conflicto vasco-navarro, en una situación post-ETA en Navarra. Máster Universitario en Dinámicas de Cambio en las Sociedades Modernas Avanzadas. Recuperado de https://academicae.unavarra.es/handle/2454/14490.

Clavijo, G. A. C. (2010). La universidad colombiana de cara al 2050. Teoría y praxis investigativa, 5(1), 43-58. Recuperado de file://D:/Downloads/Dialnet-LaUniversidadColombianaDeCaraAl2050-3701451.pdf

Cruz, A. F. (2008). Educar para gestionar conflictos en una sociedad fragmentada. Una propuesta educativa para una cultura de paz. (Tesis doctoral). Departamento de Teoría e Historia de la educación, Universidad de Barcelona. España. Recuperado de http://www.tdx.cat/bitstream/handle/10803/2935/FCA_TESIS.pdf;jsessionid=1F3574F6E3366E86B97A6A7 $1 \mathrm{AC67271C}$ ?sequence $=1$ 
Fajardo Pascagaza, E. (2016). Hacia la caracterización de los valores democráticos y ciudadanos de los estudiantes universitarios: una mirada desde la formación política y la construcción de escenarios de paz. Revista Interamericana de Educación, Pedagogía y Estudios Culturales, 9(1), 87-105. Recuperado de file://D:/Downloads/3604-10707-1-PB\%20(1).pdf

Franco, M., Bautista, F., \& Carmona, M. (2009). Propuesta de educación y cultura de paz para la ciudad de Puebla (México). (Tesis doctoral), Instituto de la paz y el conflicto, Universidad de Granada.Recuperado de http://www.ugr.es/ erivera/PaginaDocencia/Posgrado/Documentos/AbregoGuadalupe.pdf

Godet, M., y Vásquez, J. M. (2011). Prospectiva y estrategia: enfoques integrados. Cuadernos de Administración, 14(21), 165-176. Recuperado de https://cuadernosdeadministracion.univalle.edu.co/index.php/cuadernos_de_administracion/article/view /112.

González Rubio. A. (2007). Incidencia de cooperación internacional en política pública: casos de la política para la paz en Colombia. Política de educación para la paz en Colombia. Disponible en línea: file:///D:/Downloads/Cooperaci_n_internacional_y_pol_tica_p_blica.pdf

Herrera, C. J. (2009). Reconciliaciones, transiciones democráticas y construcción de paz. El caso colombiano 1989-2006. Recuperado de https://digibug.ugr.es/bitstream/handle/10481/2165/17826196.pdf?sequence=1\&isAllowed=y

Infante Márquez, A. (2014). El papel de la educación en situaciones de posconflicto: estrategias y recomendaciones. Hallazgos, 11(21). Recuperado de http://revistas.usantotomas.edu.co/index.php/hallazgos/article/view/1156

Jiménez, S. (2012). La administración de los efectos de la guerra como tecnología de gobierno: una mirada postliberal al conflicto armado en Colombia, 1980-2010. (Serie Tesis Doctorado) ISBN: 978-9978-67-416-1. Recuperado de http://biblio.flacsoandes.edu.ec/catalog/resGet.php?resld=54474

Jiménez, F. \& Jiménez, F. (2014). Una historia de la investigación para la paz. Historia Actual Online, 34, 149162.Recuperado de https://dialnet.unirioja.es/servlet/articulo?codigo=4851747.

Katz, M. (2011). Construcción de paz: una aproximación desde las capacidades locales (Doctoral dissertation, Universidad Nacional de Colombia). Recuperado de http://www.bivipas.unal.edu.co/handle/10720/588

Loaiza, A. (2012). Negociaciones de paz en Colombia, 1982-2009. Un estado del arte. Estudios políticos, (40), 175-200. Recuperado de http://www.iatreia.udea.edu.co/index.php/estudiospoliticos/article/view/13210.

Machado, A. L. (2011). La educación en América Latina y el Caribe: Visión prospectiva al año 2020. Recuperado de http://www. reggen. org. br/midia/documentos/laeducacionenamericalatina. Pdf

Marín Arias. V.; García Gonzales, N.; \& Buriticá Valencia, V. (2013). Hacia la construcción del estado del arte sobre sentidos y prácticas de los y las jóvenes en Colombia, relacionados con acción política, construcción de paz y no violencia.

Mateos, R. (2012). La construcción de paz posbélica. Análisis de los debates críticos a través del caso de Sierra leona. (Tesis doctoral), doctorado de relaciones internacionales e integración Europea, Universidad Autónoma de Barcelona. España. Recuperado de http://www.tdx.cat/bitstream/handle/10803/96422/omm1de1.pdf?sequence=1

Montes, C. B. (2014). Desafíos y estado futuro de la convivencia en Colombia al 2025. Criminalidad, 56(2), 319332.Recuperado de https://policia.gov.co/sites/default/files/56209.html. 
Muñoz, J. M. (2014). Planificación por escenarios: preparando pequeñas empresas del comercio minorista de muebles planeados para un futuro competitivo. (Tesis de Maestría) Maestría en Administración. Universidad Nacional de Colombia.

Mur Barinas, D. A., \& Contreras Clavijo, J. C. (2019). Los imaginarios de paz de maestros en formación de la universidad de La Salle. Retrieved from https://ciencia.lasalle.edu.co/lic_lenguas/875.

Muslera, E. A. R. (2016). El proceso de construcción de paz colombiano más allá de la negociación: una propuesta desde la Paz Transformadora y Participativa. El Ágora USB, 16(2), 513-532. Recuperado de http://www.redalyc.org/pdf/4077/407755354009.pdf

Navarro, I.; Royo, J. M.; Urgell, J.; Urrutia, P.; Villellas, A. \& Villellas, M. (2016). Oportunidades de paz y escenarios de riesgo para 2017. Informe. escola de cultura de pau. España. Recuperado de http://escolapau.uab.cat/img/programas/alerta/alerta/17/OportunidadesyRiesgos2017.pdf

Nasi, C., y Rettberg, A. (2005). Los estudios sobre conflicto armado y paz: un campo en evolución permanente. Colombia internacional, (62), 64-85. Recuperado http://www.scielo.org.co/pdf/rci/n62/n62a05.pdf

Ninkov, M. L. (2013). Los proyectos de Paz y Desarrollo y Laboratorios de Paz y la estrategia de Acción Integral como modelos de construcción de paz en Colombia. Los casos del Magdalena Medio, el Oriente Antioqueño y La Macarena. Program of Development Management \& Policy; Washington, Estado Unidos. Recuperado de https://repository.library.georgetown.edu/handle/10822/558256

Olave, G. (2013). El proceso de paz en Colombia según el Estado y las FARC-EP. Discurso \& sociedad, 7(2), 338363. Recuperado de https://dialnet.unirioja.es/servlet/articulo?codigo=4649014

Padilla, H. (2017). Carismas en la universidad para la construcción de la paz. Revista de La Universidad de La Salle, (72), 83-101.Recuperdo de https://ciencia.lasalle.edu.co/ruls/vol2017/iss72/6/.

Parra Valencia, L. M. (2014). Prácticas y experiencias colectivas ante la guerra y para la construcción de paz: Iniciativas sociales de paz en Colombia. El Ágora USB, 14(2), 377-395. Recuperado de https://dialnet.unirioja.es/servlet/articulo?codigo=5372947

Peña, G. J. (2014). Multinacionales y responsabilidad social empresarial en la construcción de paz en Colombia. Cuadernos de administración, 27(48), 67-96. Recuperado de https://www.redalyc.org/pdf/205/20531183004.pdf

Peña, G. (2015). El rol de las Instituciones de Educación Superior (IES) frente al posconflicto 2014-2015. Bogotá: Universidad Militar Nueva Granada. Recuperado de https://repository.unimilitar.edu.co/handle/10654/13763

Peláez, M. M., Álvarez, Y. A. A., Palacio, I. C. A., \& Mazo, A. Z. (2017). Aplicación de los ejes de Schwartz como metodología de prospectiva tecnológica al modelo universitario-empresa en el contexto colombiano. Revista Ingenierías USBMed, 8(1), 63-70. https://doi.org/10.21500/20275846.2772

Pérez, T. H. (2011). Conflicto y posconflicto en Colombia: una mirada a la política de seguridad democrática. Magistro, 5(10), 129-150. Recuperado de http://revistas.usta.edu.co/index.php/magistro/article/view/553.

Pérez, T. H. (2015). Colombia: de la educación en emergencia hacia una educación para el posconflicto y la paz. Revista Interamericana de Investigación, Educación y Pedagogía, RIIEP, 7(2), 287-311. Recuperado de http://revistas.usantotomas.edu.co/index.php/riiep/article/viewFile/1959/2069

PNUD (2019). CAQUETA: Retos y desafíos para el Desarrollo Sostenible. Programa de las Naciones Unidas para el Desarrollo. Recuperado de 
https://www.co.undp.org/content/colombia/es/home/library/democratic_governance/caqueta_--retos-ydesafios-para-el-desarrollo-sostenible.html

Prieto, E. (2017). Análisis sobre la viabilidad industrial en el escenario de postconflicto. (Tesis de posgrado), Universidad Militar Nueva Granada. Colombia. Recuperado de https://repository.unimilitar.edu.co/bitstream/handle/10654/15818/PrietoSanchezElkinRicardo2017.pdf? sequence $=2$.

Rettberg, A. (2010). En busca de socios: Canadá, iniciativas empresariales y la construcción de paz en Colombia. Perspectivas Colombo Canadienses, 1(1). Recuperado de http://revistas.urosario.edu.co/index.php/perspectiva/article/view/903.

Rettberg, A. (2013). La construcción de paz bajo la lupa: una revisión de la actividad y de la literatura académica internacional. Estudios Políticos, (42), 13-36. Recuperado de http://www.scielo.org.co/scielo.php?script=sci_abstract\&pid=S0121-51672013000100002

Rettberg, A., Arias Rodríguez, M. A., Ardila, B., Bastidas, L., Guzmán, M., Pinzón, J. S., ... \& Vargas, M. G. (2017). 1900 iniciativas de paz en Colombia: caracterización y análisis de las iniciativas de paz de la sociedad civil en Colombia 1985-2016.

https://pasocolombia.org/sites/default/files/1900_iniciativas_de_paz_nacional.pdf.

Rey, F. G. (2010). Organización territorial, desarrollo sostenible y nuevas visiones sobre el territorio en Colombia (1991-2010). Perspectiva geográfica, (15), 239-260. Recuperado de https://papers.ssrn.com/sol3/papers.cfm?abstract_id=2455866.

Rivero, J. L. A. (2017). Las alianzas estratégicas y sus singularidades en las relaciones de las instituciones de educación superior y las empresas. Estrategia y Gestión Universitaria, 3(2), 56-67. Recuperado de http://revistas.unica.cu/index.php/regu/article/view/366

Rodríguez, A. (2013). Elementos de construcción de paz en los programas musicales colectivos Una aproximación al programa Música para la Reconciliación de la Fundación Nacional Batuta en Colombia.

Rosanía, N. A. (2014). La construcción de la paz en Colombia: desafíos desde la Escola de cultura de Pau de Barcelona y la ONU. (Ensayo de proyecto de investigación), Universidad Militar Nueva Granada. Colombia, recuperado de http://repository.unimilitar.edu.co/handle/10654/11524

Salamanca, M. (Ed.). (2008). Las prácticas de la resolución de conflictos en América Latina (Vol. 15). Universidad de Deusto. Recuperado de https://dialnet.unirioja.es/servlet/libro?codigo=378283.

Sánchez, M. (2010). La educación para la paz en Colombia: una responsabilidad del Estado Social de Derecho. Revista Vía luris, (9), 141-160. Recuperado de https://dialnet.unirioja.es/servlet/articulo?codigo=3432200

Sánchez. A.D.P. (2013). Los programas musicales colectivos como espacios de construcción de paz. Caso Programa Música para la Reconciliación de la Fundación Nacional Batuta en Colombia. Fòrum de Recerca, (18), 81-96. Recuperado de http://repositori.uji.es/xmlui/bitstream/handle/10234/74407/TFM_Andrea\%20Rodr_guez.pdf?sequence=1

Santamaría, M. L., \& Grueso, M. P. (2019). Empresa, empresarios y construcción de paz en Colombia: hallazgos y retos. Revista de ciencias sociales, 25(1), 146-155.

Salguero, J. Seva,M. (2005). Educar para la paz: el caso de un país dominado por la violencia: Colombia (Doctoral dissertation, Tesis (Doctor en ciencias de la Educación). Madrid: Universidad Complutense de Madrid. Facultad de Educación. Departamento de Didáctica y Organización Escolar.(Versión electrónica). Recuperado de http://eprints. ucm. es/tesis/edu/ucm-t27290. Pfd. 
Sarti, C. (2007). Construcción de paz y resolución de conflictos. Construction de la paix et résolution de conflits. Irenes. Ciudad del Carmen, Campeche, México. Recuperado de: http://www. irenees. net/bdf_ficheanalyse-782_es. html.

Silva, B. (2017). Processos de paz na Colômbia: dos intentos de Andrés Pastrana à instalação da Mesa de Havana. Relações Internacionais (UNESP - UNICAMP - PUC-SP) - FFC, Brasil. Recuperado de https://repositorio.unesp.br/handle/11449/150590.

Smith Jr, D. (2017). Un nuevo modelo de construcción de paz. Cultura de Paz, 23(71). Recuperado en: https://www.revistasnicaragua.net.ni/index.php/culturadepaz/article/view/3023. Cultura de Paz. Managua, Nicaragua • Volumen 23・ $\mathrm{N}^{\circ} 71 \bullet$ Enero - Abril, 2017.

Tercero, M. A. C. (2016). Economía criminal y riesgo empresarial tras el proceso de paz en Colombia. bie3: Boletín IEEE, (3), 308-329.. Recuperado de http://www.ieee.es/Galerias/fichero/docs_opinion/2016/DIEEEO742016_EconomiaCriminal_Colombia_CumplidoTercero.pdf.

Torrermorell, M., Valls, M., De Nicolás, M., \& Raga, L. (2014). La cultura de paz en la educación para la ciudadanía y los derechos humanos en los libros de texto de educación primaria. Perfiles educativos, 36(146), 80-97. Recuperado de https://www.sciencedirect.com/science/article/pii/S0185269814701291.

Valencia, J. A. V., Benjumea, M. L. B., Pelaéz, L. A. C., \& Orozco, C. M. T.(2013) Opciones metodológicas para la realización de estudios de prospectiva en instituciones de educación superior methodological options for performing prospective studies in higher education. Recuperado de https://www.researchgate.net/publication/303988684_Opciones_metodologicas_para_la_realizacion_de _estudios_de_prospectiva_en_Instituciones_de_Educacion_Superior

Valenzuela, B.; Guillén, M.; Medina, A. \& Rodríguez, P. (2017).Educación y Universidad ante el Horizonte 2020. Inclusión y cultura colaborativa entre empresa y sociedad. Universidad de Sonora, Qartuppi, S. de R.L. de C.V. ISBN 978-607-518-225-4. Recuperado de http://www.qartuppi.com/2017/HORIZONTE_2.pdf

Vargas, G. (2014). La responsabilidad social empresarial en la construcción de paz: una introducción. Universidad de los Andes. CIDER. Recuperado de http://biblioteca.clacso.edu.ar/Colombia/cider-uniandes/20170727042633/pdf_516.pdf

Villa, J. (2016). Perdón y reconciliación: una perspectiva psicosocial desde la no violencia. Polis. Revista Latinoamericana, (43). Recuperado de https://journals.openedition.org/polis/11553.

Villamarin, L. (2016). La responsabilidad social empresarial una herramienta hacia la paz en Colombia (Master's thesis, Facultad de Ciencias Políticas y Relaciones Internacionales). Recuperado de https://repository.javeriana.edu.co/handle/10554/18980.

Esta obra está bajo una Licencia Creative Commons Attribución-NoCommercial 4.0 International

(cc) BY-NC 